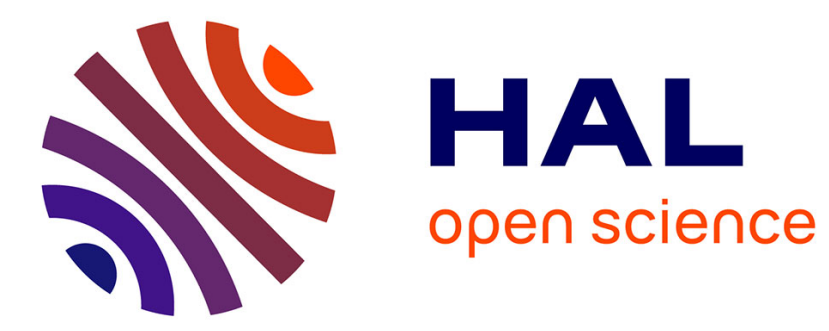

\title{
Quantitative Error Analysis for the Reconstruction of Derivatives
}

Laurent Condat, Torsten Möller

\section{To cite this version:}

Laurent Condat, Torsten Möller. Quantitative Error Analysis for the Reconstruction of Derivatives. IEEE Transactions on Signal Processing, 2011, 59 (6), pp. 2965 - 2969. 10.1109/TSP.2011.2119316 . hal-00462203v2

\section{HAL Id: hal-00462203 \\ https://hal.science/hal-00462203v2}

Submitted on 21 Jul 2010

HAL is a multi-disciplinary open access archive for the deposit and dissemination of scientific research documents, whether they are published or not. The documents may come from teaching and research institutions in France or abroad, or from public or private research centers.
L'archive ouverte pluridisciplinaire HAL, est destinée au dépôt et à la diffusion de documents scientifiques de niveau recherche, publiés ou non, émanant des établissements d'enseignement et de recherche français ou étrangers, des laboratoires publics ou privés. 


\title{
Quantitative Error Analysis for the Reconstruction of Derivatives
}

\author{
Laurent Condat and Torsten Möller
}

\begin{abstract}
We present a general Fourier-based method which provides an accurate prediction of the approximation error, when the derivative of a signal $s(t)$ is continuously reconstructed from uniform point samples or generalized measurements on $s$. This formalism applies to a wide class of convolution-based techniques. It provides a key tool, the frequency error kernel, for designing computationally efficient reconstruction schemes which are near optimal in the least-squares sense.
\end{abstract}

Index Terms-derivatives, reconstruction, sampling, interpolation, approximation, error analysis, frequency error kernel.

\section{INTRODUCTION}

$\mathbf{T}$ HE representation of a digital signal by means of a discrete/continuous model is essential for common tasks such as interpolation and resampling. For images and other two-dimensional (2-D) data, polynomial spline models based on B-splines are particularly popular, mainly due to their simplicity and excellent approximation capabilities [1].

Reconstruction of a continuous function and its derivatives from a set of samples is one of the fundamental operations in signal processing, numerical analysis, and many other fields. In visualization, for instance, the gradient is employed in volume classification and shading [2]. It has to be evaluated at arbitrary locations and not only at the discrete points where the underlying signal has been sampled. Edge detection, segmentation, motion estimation and super-resolution are other applications where partial derivatives may be required at subpixel resolution.

\section{A. Motivation}

We denote by $s(t) \in L^{2}(\mathbb{R})$ a continuously defined function (the signal) which is prefiltered and sampled at uniform locations to yield the discrete measurements

$$
u[k]=\int_{\mathbb{R}} s(t) \tilde{\varphi}\left(k-\frac{t}{T}\right) d \frac{t}{T} \quad \forall k \in \mathbb{Z},
$$

where $T$ is the sampling step and the analysis function $\tilde{\varphi}(t)$ is, for instance, the impulse response of the acquisition device. This generalized sampling scenario encompasses the case where ideal point samples $u[k]=s(T k)$ are available, simply by letting $\tilde{\varphi}(t)$ be the Dirac distribution $\delta(t)$.

The signal $s(t)$ is unknown and the sequence $u=(u[k])_{k \in \mathbb{Z}}$ represents the only available data. We are interested in constructing from $u$ an estimate of the $N$-th derivative $s^{(N)}(t)$ of

L. Condat is with the Image Team of the GREYC laboratory, a joint CNRS-UCBN-ENSICAEN research unit in Caen, France. Contact: see http://www.greyc.ensicaen.fr//lcondat/. T. Möller is with the GrUVi Lab of the Simon Fraser University (SFU), Vancouver, CA. Contact: see http://www.cs.sfu.ca/ torsten/.

This work was initiated during the visit of L. Condat at SFU, supported by a fellowship of the Computer Science Department of SFU and the NSERC. $s(t)$, for some integer $N \geq 1$. We look for a reconstruction in a linear shift-invariant space $\mathcal{V}_{T}(\varphi)=\operatorname{Span}\left(\left\{\varphi\left(\frac{t}{T}-k\right)\right\}_{k \in \mathbb{Z}}\right)$ generated by the translates of a template function $\varphi(t) \in$ $L^{2}(\mathbb{R})$ :

$$
f(t)=\frac{1}{T^{N}} \sum_{k \in \mathbb{Z}} c[k] \varphi\left(\frac{t}{T}-k\right) \quad \forall t \in \mathbb{R},
$$

where the coefficients $c[k]$ are obtained by discrete filtering with the stable prefilter $p \in \ell^{1}$ :

$$
c[k]=(u * p)[k] \quad \forall k \in \mathbb{Z} .
$$

Using this general recipe for reconstruction, we denote by $f_{\text {app }}$ an estimate of $s$ (with $N=0$ in (2)), while an estimate of the derivative $s^{(N)}$ is denoted by $f_{\text {der }}$. We remark that the reconstruction method involves a discrete prefiltering step followed by the fit of the continuous model itself. In practical applications, the prefiltering step is performed once. Its computation time is negligible in comparison with the many calls to (2) to evaluate $f$ at the desired locations.

Estimating $s(t)$ itself is the classical problem of interpolation, for which there is a vast amount of literature; see e.g. the survey papers [3]-[6] and some recent developments [7][9]. Of course, once an estimate $f_{\text {app }}(t)$ of $s(t)$ has been reconstructed, one can consider its derivative $f_{\text {app }}^{(N)}(t)$ as a valid estimate of $s^{(N)}(t)$. But there is no a priori guarantee that whenever $f_{\text {app }}$ is close to $s$ in the least-squares sense, then $f_{\text {app }}^{(N)}$ is close to $s^{(N)}$. Moreover, since efficiency considerations generally steer the design of the method, one may be interested in deriving direct estimation schemes of $s^{(N)}$, without the conceptual intermediary step of evaluating $s$, which unnecessarily constrains the conditions on accuracy and smoothness. The aim of this work is to provide a way to quantify the error between $s^{(N)}$ and its estimate $f_{\text {der }}$, so that the design of reconstruction schemes minimizing this error is made easy.

\section{B. Related Work}

There is a vast literature on designing so-called digital differentiators, which are digital filters estimating the derivative at the grid points $T k$ only, see e.g. [10] and references therein. In [11], point-wise estimates of the derivative are derived, which are optimal in the minimax sense. By contrast, we consider the context in which the derivative is reconstructed continuously in a shift-invariant space, so that it can be evaluated at every arbitrary location.

Shannon's theory provides an exact way to recover a bandlimited signal from its samples, using the sinc interpolator. Similarly, the "ideal" derivative reconstruction filter was 
shown in [12] to be the derivative of the sinc. However, its slow decay and the ringing artifacts it may introduce, prevent its practical use. Moreover, for non-bandlimited signals, the sinc-based theory is not valid any more [4], [13], [14]. That is why practitioners rely on convolutions with more localized kernels having compact support, like windowed sinc [15], [16], splines and other piecewise polynomial functions [3], [12]. In none of these works, there is an analytic comparison of different filters nor a quantitative analysis of the estimation error

In [17], the second author and its collaborators developed tools and derived absolute error bounds for the spatial analysis of both interpolation and derivative filters of arbitrary order. Approximation theory also provides a general framework, which focuses on the asymptotic error behavior of the reconstruction method as $T \rightarrow 0$ [18], [19]. These qualitative error bounds are generally not sharp enough to be of direct use to practitioners and accurate ways of predicting the approximation error are desirable, so that algorithms can be compared and the parameters $\varphi, p, \tilde{\varphi}, T$ can be chosen appropriately. For this, Blu et al. proposed a remarkable Fourier-based method which provides an accurate estimate of the approximation error, with a wide range of applicability [19]. Their approach makes the design of reconstruction algorithms simple and accurate, and it is at the heart of recent developments in interpolation theory [7]-[9]. In this paper, we extend this theory to the setting of derivative reconstruction.

\section{Notations and Paper Organization}

The Fourier transform of a function $f(t)$ is denoted by $\hat{f}(\omega)=\int_{\mathbb{R}} f(t) e^{-j \omega t} d t$. We require $\tilde{\varphi}$ in (1) to have a welldefined bounded Fourier transform.

The $\mathcal{Z}$-transform of a discrete signal $v=(v[k])_{k \in \mathbb{Z}}$ is $V(z)=\sum_{\mathbb{Z}} v[k] z^{-k}$ and its Fourier transform is $\hat{v}(\omega)=$ $V\left(e^{j \omega}\right)$.

For any real $r>0$, the Sobolev space $W_{2}^{r}$ is the set of functions $f$ such that $\int_{\mathbb{R}}\left(1+\omega^{2}\right)^{r}|\hat{f}(\omega)|^{2} d \omega<\infty$. Therefore, the Sobolev regularity of $f$ is the maximum value of $r$ such that $f \in W_{2}^{r}$.

$\mathcal{P}^{n}$ is the space of polynomial functions of degree at most $n \in \mathbb{N}$. We define the causal B-spline $\beta_{+}^{n}(t)$ of degree $n \in \mathbb{N}$ by $\beta_{+}^{0}=1_{[0,1)}$ and $\beta_{+}^{n}=\beta_{+}^{n-1} * \beta_{+}^{0}$. The centered B-spline of degree $n$ is $\beta^{n}(t)=\beta_{+}^{n}\left(t+\frac{n+1}{2}\right)$.

We introduce the dual $\phi_{d}$ of a function $\phi \in L^{2}(\mathbb{R})$ by $\widehat{\phi}_{d}(\omega)=\hat{\phi}(\omega)^{*} / \hat{a}_{\phi}(\omega)$, where the discrete autocorrelation filter $a_{\phi}$ is defined by $a_{\phi}[k]=\int_{\mathbb{R}} \phi(t) \phi(t-k) d t$ and the star is for complex conjugation.

The different functions and filters used throughout the paper are illustrated by the flowgraph in Fig. 1.

The outline of this paper is as follows. In Sect. II, we introduce the frequency error kernel, which is the cornerstone for quantifying the error between a function and its estimate from discrete measurements. We present our main results in Sect. III, based on a new error kernel dedicated to the reconstruction of derivatives. In Sect. IV, we discuss the consequences of the formalism for the design of efficient

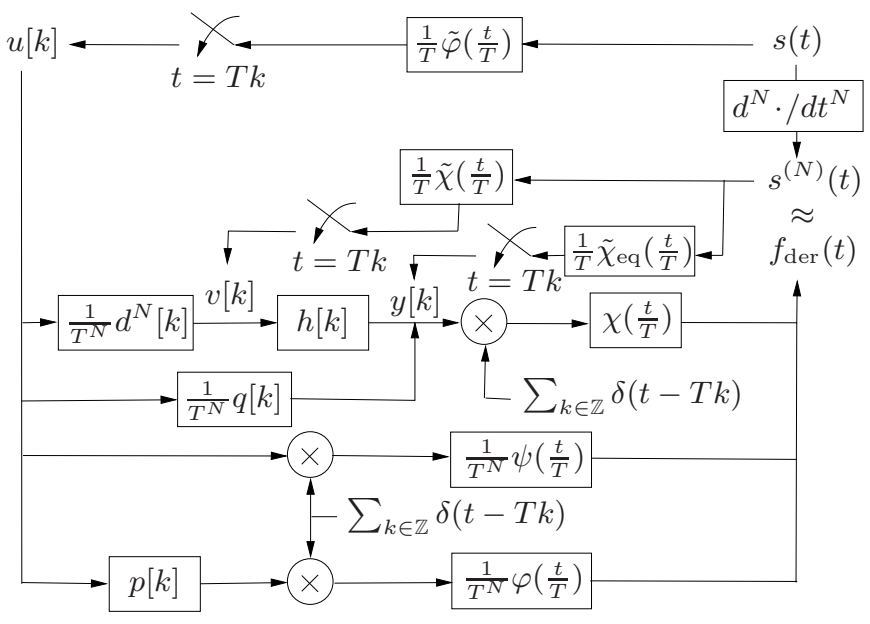

Fig. 1. Flowgraph of the different equivalent procedures for the sampling process $s \mapsto u$ and the derivative reconstruction process $u \mapsto f_{\text {der }}$.

reconstruction methods. Finally, in Sect. V, we illustrate our methodology by the study of methods reconstructing the second derivative.

\section{The Frequency ERror Kernel}

An important result of Blu et al. is that the error $\| s-$ $f_{\text {app }} \|_{L^{2}}$ between $s$ and its estimate reconstructed using (2) can be predicted very accurately by the estimate [19]:

$$
\eta_{s}(T)=\sqrt{\frac{1}{2 \pi} \int_{\mathbb{R}}|\hat{s}(\omega)|^{2} E(T \omega) d \omega},
$$

using the frequency error kernel defined by

$$
E(\omega)=\underbrace{1-\frac{|\hat{\varphi}(\omega)|^{2}}{\hat{a}_{\varphi}(\omega)}}_{E_{\min }(\omega)}+\underbrace{\hat{a}_{\varphi}(\omega)\left|\widehat{\tilde{\varphi}}(\omega) \hat{p}(\omega)-\widehat{\varphi}_{d}(\omega)\right|^{2}}_{E_{\mathrm{res}}(\omega)} .
$$

Remarkable properties of the global error estimate $\eta_{s}(T)$ include its exactness for bandlimited signals and for the average of the true approximation error over all possible shifts of the input function $s$. In the general case, we have the approximation $\left\|s-f_{\text {app }}\right\|_{L^{2}}=\eta_{s}(T)+o\left(T^{r}\right)$, assuming that $s$ has Sobolev regularity $r>\frac{1}{2}$. Moreover, in a stochastic framework where $s$ is a realization of a random stationary process instead of a deterministic function of $L^{2}, \eta_{s}(T)$ is the exact expression of the time averaged expectation of the quadratic pointwise error, by replacing the energy $|\hat{s}(\omega)|^{2}$ by the power spectrum density $\hat{c}_{s}(\omega)$ in (4). These properties and several others are detailed in [19] and [20].

In practical situations, $|\hat{s}(\omega)|^{2}$ or $\hat{c}_{s}(\omega)$ is unknown, but the multiplicative form in the integral (4) ensures that the error is small if $E(\omega)$ is close to zero. Hence, the frequency error kernel is a tool of choice for characterizing a reconstruction scheme. More precisely, the value $E(\omega)$ at a given frequency $\omega$ can be interpreted as the average power of the approximation error, in case $s(t)$ is the pure unit sinusoid $e^{j \omega t / T}[19$, Thm. $3]$. Therefore, the study of $E(\omega)$ allows to characterize the behavior of a reconstruction method at different frequency components. For instance, $E(\omega)$ for $\omega$ close to $\pi$ indicates to 
which extent the salient features, fine details and textures are preserved and aliasing is enhanced. By contrast, an asymptotic study for $\omega$ around 0 characterizes the reconstruction quality for the low frequency content of the signal $s$.

Thus, $\varphi$ and $p$ can be tuned to minimize the error kernel, so that the reconstruction quality is improved for virtually every function $s$ [7]-[9], [21]. Given the reconstruction space $\mathcal{V}_{T}(\varphi)$, the error kernel $E(\omega)$ attains its minimum value $E_{\min }(\omega)$, for every $\omega \in \mathbb{R}$, when $f_{\text {app }}$ is the minimum error reconstruction of $s$ in $\mathcal{V}_{T}(\varphi)$; that is, its orthogonal projection onto $\mathcal{V}_{T}(\varphi)$. Thus, the prefilter $p$ should be designed so that $E(\omega)$ is close to $E_{\text {min }}(\omega)$, in order for the method to behave like this optimal, but generally unattainable, least-squares approximation [9], [19].

\section{Computation of the ERror Estimates}

We first define the function $\psi(t)$ by

$$
\psi(t)=\sum_{k \in \mathbb{Z}} p[k] \varphi(t-k) \Leftrightarrow \hat{\psi}(\omega)=\hat{p}(\omega) \hat{\varphi}(\omega) .
$$

Then, $f_{\text {der }}(t)=\frac{1}{T^{N}} \sum_{k \in \mathbb{Z}} u[k] \psi\left(\frac{t}{T}-k\right)$. Although in practice $\varphi$ is chosen with compact support, so that (2) is computationally attractive, the function $\psi$, which is the impulse response of the reconstruction operation, can have infinite support.

We assume that the following equivalent conditions on $\psi$ are satisfied, so that $f_{\text {der }}$ does not blow up as $T$ tends to zero (because of the $1 / T^{N}$ factor in (2)):

$$
\begin{aligned}
& f_{\text {der }}=0 \text { if } s \in \mathcal{P}^{N-1}, \\
\Leftrightarrow & \sum_{k \in \mathbb{Z}} P(k) \psi(t-k)=0, \quad \forall t \in \mathbb{R}, \forall P \in \mathcal{P}^{N-1}, \\
\Leftrightarrow & \widehat{\psi}^{(n)}(2 k \pi)=0, \quad \forall k \in \mathbb{Z}, \forall n=0 \ldots N-1 .
\end{aligned}
$$

Although these equivalences may be classical in harmonic analysis, we rederive them in the Appendix A for sake of completeness.

Our second requirement is that $\psi$ can be decomposed as

$$
\psi(t)=\sum_{k \in \mathbb{Z}} q[k] \chi(t-k) \Leftrightarrow \hat{\psi}(\omega)=\hat{q}(\omega) \hat{\chi}(\omega),
$$

where $q \in \ell^{1}$ is a discrete filter and the integer translates of the function $\chi \in L^{2}$ form a Riesz basis; that is, there exist two constants $B \geq A>0$ such that $A \leq \hat{a}_{\chi}(\omega) \leq B$ almost everywhere. Thus, each function of the reconstruction space $\mathcal{V}_{T}(\chi)$ has a unique and stable expansion in terms of the shifts of $\chi$. Note that this condition is not restrictive; in particular, there is no requirement that $\chi$ be compactly supported, even if $\varphi$ is.

Our last requirement is that $s \in L^{2}$ has Sobolev regularity $r>N+\frac{1}{2}$, so that $s$ has at least $N$ continuous derivatives in $L^{2}$.

The Riesz basis condition together with the requirement (9) imply that 0 is a root of $q$ of multiplicity $N$. In other words, there exists a stable filter $h \in \ell^{1}$ such that $q=h * d^{N}$, where the iterated causal finite difference filter $d^{N}$ is defined by $D^{N}(z)=\left(1-z^{-1}\right)^{N}$. Thus, we essentially decomposed $\psi=p * \varphi$ into a moving difference filter $q$ and a well posed reconstruction kernel $\chi$. This allows us to recast derivative

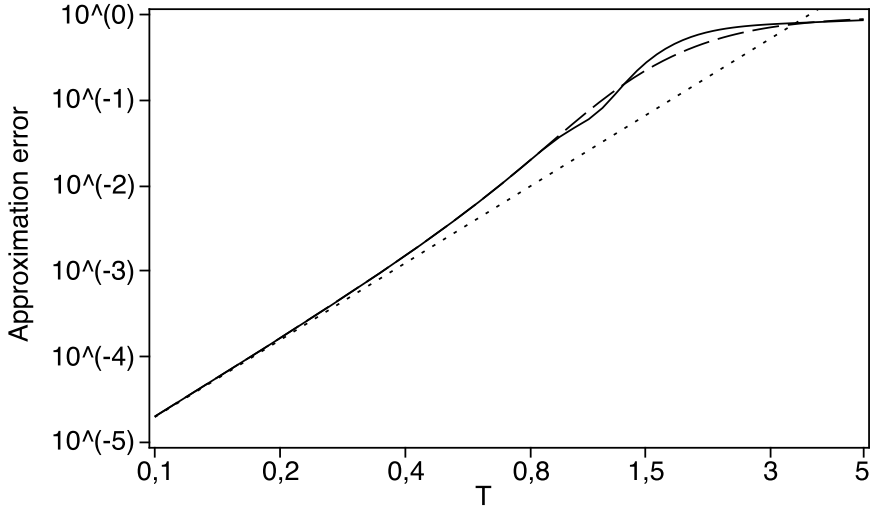

Fig. 2. Approximation error as a function of the sampling step $T$. We estimate the first derivative $s^{\prime}(t)$ of $s(t)=e^{-\frac{(t-1)^{2}}{2}}$ by the first derivative of the cubic spline interpolating the point samples $u[k]=s(T k)$. Thus, we have $\tilde{\varphi}=\delta, \varphi=\left(\beta^{3}\right)^{\prime}$ et $P(z)=6 /\left(z+4+z^{-1}\right)$. The error estimate $\eta_{s^{\prime}}(T)$ (dashed line) is close to the true error $\left\|s^{\prime}-f_{\text {der }}\right\|_{L^{2}}$ (solid line). The dotted line is the asymptote $C\left\|s^{(4)}\right\|_{L^{2}} T^{3}$, where the asymptotic constant $C=1 / \sqrt{30240}$ is obtained from the Taylor development $E(\omega)^{1 / 2} \sim C \omega^{3}$.

reconstruction into the framework summarized in Sect. II, by reasoning on $\chi$ instead of $\varphi$. For this, we need the following property:

Lemma. We define $v=\frac{1}{T^{N}} u * d^{N}$. Then, $v[k]=$ $\int_{\mathbb{R}} s^{(N)}(t) \tilde{\chi}\left(k-\frac{t}{T}\right) d \frac{t}{T}$, where $\tilde{\chi}=\tilde{\varphi} * \beta_{+}^{N-1}$.

This important lemma, proved in Appendix B, allows us to interpret the filtered samples $u[k]$ as generalized samples of the derivative $s^{(N)}$. Hence, we now have all the ingredients to formulate the following results, proved in Appendix B.

Theorem 1. $\left\|s^{(N)}-f_{\text {der }}\right\|_{L^{2}}=\eta_{s^{(N)}}(T)+o\left(T^{r-N}\right)$, where

$$
\eta_{s^{(N)}}(T)=(\frac{1}{2 \pi} \int_{\mathbb{R}} \underbrace{|\hat{s}(\omega)|^{2} w^{2 N}}_{\left|\widehat{s^{(N)}}(\omega)\right|^{2}} E(T \omega) d \omega)^{1 / 2}
$$

and the new frequency error kernel characterizing derivative reconstruction is

$$
E(\omega)=\underbrace{1-\frac{|\widehat{\varphi}(\omega)|^{2}}{\hat{a}_{\varphi}(\omega)}}_{E_{\min }(\omega)}+\underbrace{\hat{a}_{\varphi}(\omega)\left|\widehat{\tilde{\varphi}}(\omega) \hat{p}(\omega) \frac{1}{(j \omega)^{N}}-\widehat{\varphi}_{d}(\omega)\right|^{2}}_{E_{\mathrm{res}}(\omega)} .
$$

Moreover, the correction term $o\left(T^{r-N}\right)$ vanishes if $s$ is bandlimited in $\left[-\frac{\pi}{T}, \frac{\pi}{T}\right]$, or if $\tilde{\varphi}$ and $\varphi$ are both bandlimited in $[-\pi, \pi]$.

Theorem 2. In a stochastic framework where $s$ is a realization of a random stationary process with power spectrum density $\hat{c}_{s}(\omega)$, instead of a deterministic function of $L^{2}$, we have

$$
\eta_{s}(N)(T)=\left(\frac{1}{T} \int_{0}^{T} \mathcal{E}\left\{\left|s^{(N)}(t)-f_{\text {der }}(t)\right|^{2}\right\} d t\right)^{1 / 2},
$$

by replacing $|\hat{s}(\omega)|^{2}$ by $\hat{c}_{s}(\omega)$ in (11). 
In Fig. 2, we give an example of the error estimate $\eta_{s^{\prime}}(T)$ for a practical experiment in which we reconstruct the derivative of a Gaussian from point samples. This shows that $\eta_{s^{(N)}}(T)$ is an accurate, shift invariant approximation of the true error. We note that if $s \in W_{2}^{r}$ for every $r \geq 0$, which is the case in our example, then the difference between $\eta_{s^{(N)}}(T)$ and the true error decays faster than every polynomial in $T$ as $T$ tends to zero. This means that $\eta_{s^{(N)}}(T)$ can be considered as the exact value of the error in some non-infinitesimal interval; e.g. for $T \in[0,0.9]$ in Fig. 2. In the general case, $\eta_{s(N)}(T)$ is a reliable estimate of the error for practical values of $T$. By contrast, error analysis approaches based on Taylor series only apply to the asymptotic regime where $s$ is highly oversampled.

\section{As ymptotic Approximation Performance}

In this section, we focus on the reconstruction of lowpass signals; that is, we assume that $s^{(N)}$ has most of its energy around $\omega=0$. This is the case for natural images, at least for $N=1$. For other types of signals, the same study could be performed around another frequency than 0 , with exponential splines taking the role of the B-splines [22].

\section{A. The Approximation Order}

From Theorem 1, due to the closed form of $\eta_{s^{(N)}}(T)$, it is easy to expand this estimate in a power series of $T$ to obtain the exact behavior of the error as $T \rightarrow 0$. Specifically, if $s^{(N)}$ has at least Sobolev regularity $L$, we have the equivalence

$$
\begin{aligned}
& E(\omega)^{1 / 2} \sim C \omega^{L} \quad \text { as } \omega \rightarrow 0 \\
& \text { iff }\left\|s^{(N)}-f_{\text {der }}\right\|_{L^{2}} \sim C\left\|s^{(N+L)}\right\|_{L^{2}} T^{L} \quad \text { as } T \rightarrow 0 .
\end{aligned}
$$

In that case, we speak about a $L$ th-order approximation scheme. When most of the spectral energy of the signal is concentrated in the neighborhood of $\omega=0$, like with natural images, the approximation order $L$ is the most crucial determinant of the reconstruction quality and should be chosen as large as possible. To have an approximation order $L$, it is necessary that $\chi$ satisfies the Strang-Fix conditions of order $L$ [18]:

$$
\hat{\chi}(0) \neq 0 \quad \text { and } \quad \hat{\chi}^{(n)}(2 k \pi)=0 \text { for }\left\{\begin{array}{l}
k \neq 0 \\
n=0 \ldots L-1
\end{array} .\right.
$$

It was shown in [7] that a function $\chi$ with approximation $L$ has a support size $S \geq L$ with equality iff $\chi$ is a MOMS. Therefore, the reconstruction schemes having the optimal tradeoff between the reconstruction quality and the computational complexity are obtained by choosing the reconstruction kernel $\varphi$ as a MOMS.

On this point, there is an interesting connection with wavelet theory. The equivalent conditions $(7),(8),(9)$ imply that $\psi$ has $N$ vanishing moments: $\int_{\mathbb{R}} t^{n} \psi(t) d t=0, \forall n=0 \ldots N-1$. Therefore, $\psi$ is similar to a wavelet function. It is known that wavelets behave like differentiators [23]. Moreover, in [24], it is shown that there is a B-spline function at the heart of each scaling function and wavelet associated with a multiresolution analysis. In our setting, there is no such requirements like the two-scale relations associated with multiresolution. Also, the building component of $\psi$ carrying the approximation order is $\chi$. Therefore, these are the MOMS functions, a broader class than the B-splines, which naturally appear when designing $\chi$.

\section{B. The choice of the Prefilter}

We now assume that $\varphi$ is fixed. This determines $\chi$ and its approximation order $L$ (eqn. (16)). Then, we have to choose $p$ so as to exploit at best the properties of the reconstruction space $\mathcal{V}_{T}(\varphi)$; that is, so that the scheme has approximation order $L$ (eqns (14),(15)). In fact, $f_{\text {der }} \in \mathcal{V}_{T}(\varphi) \subset \mathcal{V}_{T}(\chi)$ and $p$ controls which approximation of $s^{(N)}$ in $\mathcal{V}_{T}(\varphi)$ is picked by the method. The best possible reconstruction is the orthogonal projection of $s^{(N)}$ in $\mathcal{V}_{T}(\varphi)$. The error between this optimal approximation and $s^{(N)}$ decays like $T^{L}$ and is characterized by the error kernel $E_{\min }$ given in (12). Thus, the reconstruction scheme has approximation order $L$ if and only if

$$
E(\omega)=E_{\min }(\omega)+O\left(\omega^{2 L}\right)
$$

To characterize $p$ more precisely, we define the function

$$
\tilde{\chi}_{\mathrm{eq}}(t)=\sum_{k \in \mathbb{Z}} h[k] \tilde{\chi}(t-k),
$$

where we recall that $\tilde{\chi}=\tilde{\varphi} * \beta_{+}^{N-1}$ and $\hat{p} \cdot \hat{\varphi}=\hat{h} \cdot \hat{d}^{N} \cdot \hat{\chi}$. So, we have $f_{\text {der }}(t)=\sum_{k \in \mathbb{Z}} y[k] \chi\left(\frac{t}{T}-k\right) \forall t \in \mathbb{R}$, with $y[k]=$ $\int_{\mathbb{R}} s^{(N)}(t) \tilde{\chi}_{\text {eq }}\left(k-\frac{t}{T}\right) d \frac{t}{T} \quad \forall k \in \mathbb{Z}$. Then, the reconstruction scheme has approximation order $L$ if and only if $\tilde{\chi}_{\text {eq }}$ and $\chi$ are quasi-biorthonormal with order $L$ [19]; that is,

$$
\widehat{\tilde{\chi}}_{\mathrm{eq}}(\omega) \hat{\chi}(\omega+2 k \pi)=\delta_{k}+O\left(\omega^{L}\right), \quad \forall k \in \mathbb{Z},
$$

which is equivalent for $\tilde{\chi}_{\text {eq }}$ and $\chi_{d}$ to have the same moments up to order $L$ :

$$
\int_{\mathbb{R}} t^{n} \tilde{\chi}_{\mathrm{eq}}(t) d t=\int_{\mathbb{R}} t^{n} \chi_{d}(t) d t, \quad \forall n=0 \ldots L-1 .
$$

We can express these conditions in terms of $\tilde{\varphi}, \varphi$ and $p$ as follows:

$$
\frac{\hat{p}(\omega)}{(j \omega)^{N}} \widehat{\tilde{\varphi}}(\omega) \hat{\varphi}(\omega+2 k \pi)=\delta_{k}+O\left(\omega^{L}\right), \quad \forall k \in \mathbb{Z},
$$

or, equivalently,

$$
\hat{p}(\omega) \widehat{\tilde{\varphi}}(\omega)=\frac{(j \omega)^{N}}{\hat{\varphi}(\omega)}+O\left(\omega^{L+N}\right) .
$$

We note that (22) can be obtained directly from (17).

Thus, it is preferable to choose $p$ so that these quasibiorthonormality conditions are satisfied. There is a great freedom in this respect, since only the $L+N$ linear constraints given by (22) have to be satisfied for the scheme to have the maximal approximation order, given $\varphi$.

\section{Case Study: Reconstruction of the Second DERIVATIVE}

To illustrate the benefits of the framework, we compare several methods reconstructing the second derivative from point samples $(N=2, \tilde{\varphi}=\delta)$, by means of their error kernels.

1) The first method consists in applying the finite difference filter $P(z)=z-2+z^{-1}$ to the data, then in interpolating 
the obtained sequence using the linear B-spline $\varphi=\beta^{1}$. Thus, the reconstructed function $f_{\text {der }}$ is piecewise linear and has global regularity $\mathcal{C}^{0}$.

2) The second method is similar to the first one, with cubic spline instead of linear spline interpolation. $p$ is the combination of the finite difference filter and of the interpolation prefilter [3]: $P(z)=6\left(z-2+z^{-1}\right) /(z+$ $\left.4+z^{-1}\right)$, and $\varphi=\beta^{3}$ is the cubic B-spline. Thus, the reconstructed function $f_{\text {der }}$ is piecewise cubic and has global regularity $\mathcal{C}^{2}$.

3) The third method consists in computing the second derivative of the cubic spline interpolating the samples $u[k]$. Then, we have $P(z)=6 /\left(z+4+z^{-1}\right)$ and $\varphi=\left(\beta^{3}\right)^{\prime \prime}$. Since we have the property $\left(\beta^{3}\right)^{\prime \prime}(t)=$ $\beta^{1}(t-1)-2 \beta^{1}(t-1)+\beta^{1}(t+1)$, the method can also be implemented by using $P(z)=6(z-2+$ $\left.z^{-1}\right) /\left(z+4+z^{-1}\right)$ and $\varphi=\beta^{1}$. We notice that this third method is hybrid between the two previous ones, with the generator $\varphi$ of the first method and the prefilter of the second one.

4) The last method, by $\varepsilon$-differentiation, consists in applying a finite difference to the spline $f_{a p p}(t)$ interpolating the samples $u[k]$ :

$$
f_{\mathrm{der}}(t)=\frac{1}{\varepsilon^{2}}\left(f_{a p p}(t-\varepsilon)-2 f_{a p p}(t)+f_{a p p}(t+\varepsilon)\right),
$$

for some $\varepsilon>0$. This method is particularly interesting for applications where estimates of both $s(t)$ and $s^{\prime \prime}(t)$ have to be reconstructed at the same time, for instance in volume rendering [2]. We remark that when $\varepsilon \rightarrow 0$, $f_{\text {der }}$ converges to the second derivative $f_{\text {app }}^{\prime \prime}$, which corresponds to method 3). Moreover, if $\varepsilon=1$, the method is equivalent to method 2$)$. In the general case, the method formally amounts to take $P(z)=6 /\left(z+4+z^{-1}\right)$ and $\varphi(t)=\left(\beta^{3}(t-\varepsilon)-2 \beta^{3}(t)+\beta^{3}(t+\varepsilon)\right) / \varepsilon^{2}$. The reconstructed function $f_{\text {der }}$ is a cubic spline, with global regularity $\mathcal{C}^{2}$, but with non-uniform knots. The question arises how to chose the optimal value of $\varepsilon$. We will see that the error kernel provides us with a simple way of deriving this value.

We first notice that the four methods have approximation order 2. Indeed, we have the Taylor series $E(\omega)^{1 / 2} \sim C \omega^{2}$, where the asymptotic constant $C$ is, for the first three methods, $C=\frac{\sqrt{105}}{60} \approx 0.17, C=\frac{1}{12} \approx 0.08, C=\frac{\sqrt{5}}{60} \approx 0.04$, respectively. For the fourth method, the value of $C$ depends on $\varepsilon$. For $\varepsilon \in(0,1 / 2]$, we have:

$$
C^{2}=\frac{1}{720}-\frac{1}{72} \varepsilon^{2}+\frac{31}{1260} \varepsilon^{3}-\frac{1}{180} \varepsilon^{4}
$$

and $C$ is higher for $\varepsilon>1 / 2$. Thus, we can choose $\varepsilon$ so that $C$ is minimized, which yields the optimal value

$$
\varepsilon_{o}=\frac{93}{56}-\frac{1}{56} \sqrt{4729} \approx 0.43
$$

for which $C \approx 6.10^{-4}$.

We depicted the error kernels associated to the four methods in Fig. 3, with $\varepsilon=\varepsilon_{o}$ for the method 4). We observe that the hierarchy of the methods with respect to their asymptotic

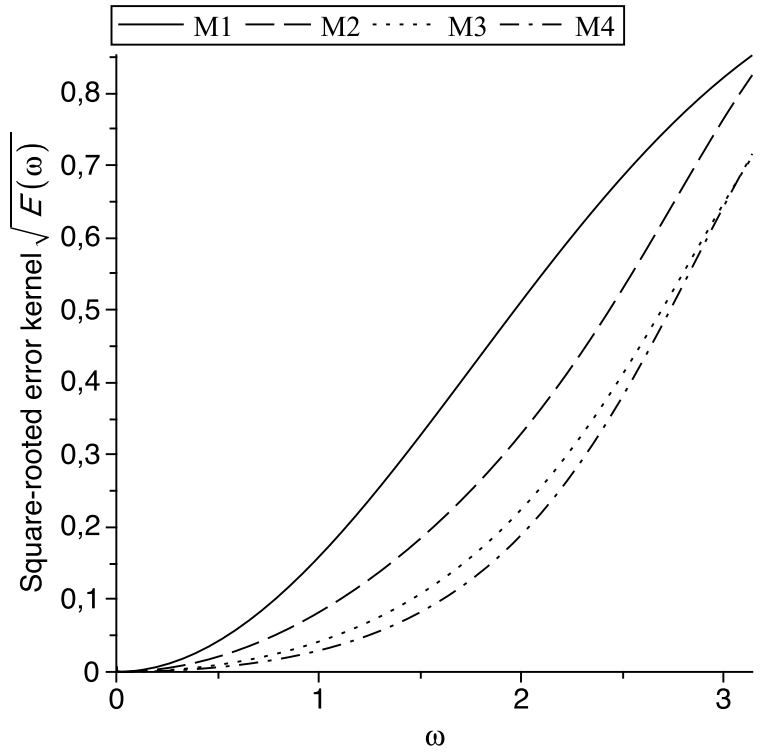

Fig. 3. Square-rooted frequency error kernel $E(\omega)^{1 / 2}$ for the reconstruction methods 1 to 4 described in Sect. V, denoted M1 to M4, respectively. Only the values in the half Nyquist band $\omega \in[0, \pi]$ are plotted, since $E(\omega)$ is symmetrical in 0 .

constants $C$ is respected. In other words, the minimization of $C$, which is an asymptotic constraint in $\omega=0$, provides error kernels whose good behavior extends in the whole Nyquist band $\omega \in[-\pi, \pi]$. This observation, which is not expected a priori, was already done for interpolation [7]-[9], [21]. Thus, the minimization of the asymptotic constant, for a given approximation order, is a simple and efficient way of designing reconstruction methods of high quality. Applying this methodology to the reconstruction of derivatives is new and the authors are not aware, for instance, of results similar to $(25)$ in the literature.

It is interesting to determine a function $\chi$ corresponding to the method 4). In fact, the translates of $\varphi$ do not form a Riesz basis. We can define $\chi$ by

$$
\hat{\chi}(\omega)=\frac{\hat{\varphi}(\omega)}{2 \cos (\omega)-2}=\frac{2 \cos (\varepsilon \omega)-2}{\varepsilon^{2}(2 \cos (\omega)-2)} \hat{\beta}^{3}(\omega),
$$

which satisfies the Strang-Fix conditions of order 2. Actually, one can show that $\chi=\beta^{1} * \beta_{\varepsilon}^{1}$ where $\beta_{\varepsilon}^{1}(t)=\frac{1}{\varepsilon} \beta^{1}\left(\frac{t}{\varepsilon}\right)$. $\chi$ has compact support in $[-1-\varepsilon, 1+\varepsilon]$, is piecewise cubic and has global regularity $\mathcal{C}^{2}$.

There is an important remark concerning method 3). We observe that $E=E_{\min }$ and, indeed, $f_{\text {der }}$ is the orthogonal projection of $s^{\prime \prime}$ in $\mathcal{V}_{T}\left(\beta^{1}\right)$. In other words, the method yields the best possible piecewise linear approximation of $s^{\prime \prime}$, although this function is unknown. More generally, it is possible to obtain the orthogonal projection of $s^{(N)}$ in the spline space $\mathcal{V}_{T}\left(\beta^{N-1}\right)$ from point samples of $s$, for every $N \geq 1$. This is a remarkable property of spline spaces.

Finally, we note that the reconstruction spaces of methods $1), 3), 4$ ) have approximation order 2 , while the cubic spline space of method 2) has approximation order 4. Hence, for method 2), the prefilter $p$ does not fully exploit the representation power of $\mathcal{V}_{T}\left(\beta^{3}\right)$. We can propose another prefilter so 
that the method has approximation order 4. After (22), this is equivalent to have

$$
\hat{p}(\omega)=-\omega^{2} \widehat{\beta}_{d}^{3}(\omega)+O\left(\omega^{6}\right)=-\omega^{2}-\frac{1}{6} \omega^{4}+O\left(\omega^{6}\right) .
$$

For instance, a solution is $P(z)=120\left(z-2+z^{-1}\right) /(66+$ $\left.26\left(z+z^{-1}\right)+z^{2}+z^{-2}\right)$ and the method amounts to compute the second derivative of the spline of degree 5 interpolating the samples $u[k]$.

\section{CONCLUSION}

We introduced a generic Fourier methodology to evaluate the quality of shift-invariant methods that continuously reconstruct the derivative of a function from discrete measurements. In our future work, we will focus on the use of this theory to design efficient reconstruction schemes [25]. We will also investigate the extension of the formalism to noisy measurements [26], [27].

Since the frequency error kernel can be defined for multidimensional signals on lattices, like in [21], the extension of this work to the evaluation of partial derivatives of multidimensional signals is straightforward [25]. Interesting applications include finite difference methods and the numerical resolution of PDEs.

\section{APPENDIX A \\ PRoOF OF THE EQUiVALENCE OF (7),(8),(9)}

We first prove that $(8) \Leftrightarrow(9)$. Let $n$ be in $0 \ldots N-1$. We define $y_{n}=\left(y_{n}[k]\right)_{k \in \mathbb{Z}}$ by $y_{n}[k]=\widehat{\psi}^{(n)}(2 k \pi)$. Then, $y_{n}[k]=0 \forall k \Leftrightarrow \widehat{y_{n}}=0 \Leftrightarrow \sum_{k}(t-k)^{n} \psi(t-k)=0 \forall t$, where the last equivalence is Poisson's sum formula applied to the function $t^{n} \psi(t)$. So, (9) $\Leftrightarrow \sum_{k \in \mathbb{Z}} Q(t-k) \psi(t-k)=$ $0, \forall t \in \mathbb{R}, \forall Q \in \mathcal{P}^{N-1}$. This is equivalent to (8) by letting, for any $t, Q(X)$ be $P(t-X)$.

We then show that $(8) \Rightarrow(7)$. We assume (8). Let $s(t)=$ $\sum_{n=0}^{N-1} a_{n} t^{n}$ be in $\mathcal{P}^{N-1}$ and $k$ be in $\mathbb{Z}$. We introduce the function $g(t)=\frac{1}{T} s(t) \tilde{\varphi}\left(k-\frac{t}{T}\right)$. Then, $u[k]=\hat{g}(0)=$ $\left.\sum_{n=0}^{N-1} a_{n} j^{n} \frac{d^{n}}{d \omega^{n}}\left\{e^{-j \omega T k} \widehat{\tilde{\varphi}}(T \omega)^{*}\right\}\right|_{\omega=0}$ is a polynomial in $k$ of degree $N-1$. Applying (8) to this polynomial $P$, we get $f_{\text {der }}=0$.

We don't give the details of $(7) \Rightarrow(8)$. This involves exhibiting, for a given $P \in \mathcal{P}^{N-1}$, a polynomial $s \in \mathcal{P}^{N-1}$ such that $u[k]=P(k)$, which is always possible.

\section{APPENDIX B \\ Proofs of THE RESUlts IN SECT. III}

We first prove the lemma. We define the function $s_{T}(t)=$ $s(T t)$. Then, for every $n$ in $1 \ldots N, s_{T}^{(n)} * \beta_{+}^{0}(t)=$ $\int_{t-1}^{t} s^{(n)}(x) d x=s_{T}^{(n-1)}(t)-s_{T}^{(n-1)}(t-1)=s_{T}^{(n-1)} *(\delta-$ $\delta(\cdot-1))(t)$. By recurrence, using the property $\beta_{+}^{n}=\beta_{+}^{n-1} * \beta_{+}^{0}$, we get $s_{T}^{(N)} * \beta_{+}^{N-1} * \tilde{\varphi}=s_{T} *(\delta-\delta(\cdot-1))^{N} * \tilde{\varphi}$. Therefore, since $u[k]=s_{T} * \tilde{\varphi}(k)$, we have $s_{T}^{(N)} * \tilde{\chi}(k)=$ $T^{N} \int_{\mathbb{R}} s^{(N)}(t) \tilde{\chi}\left(k-\frac{t}{T}\right) d \frac{t}{T}=u * d^{N}[k]$.

The theorems 1 and 2 are adaptations of [19, Theorem 1] and [19, Theorem 3], respectively, by making the substitutions $s \rightarrow s^{(N)}, u \rightarrow v, \varphi \rightarrow \chi, \tilde{\varphi} \rightarrow \tilde{\chi}, p \rightarrow h$. Thus, the theorems are satisfied with the following expression of $E(\omega)$ in (11):

$$
E(\omega)=1-\frac{|\hat{\chi}(\omega)|^{2}}{\hat{a}_{\chi}(\omega)}+\hat{a}_{\chi}(\omega)\left|\widehat{\tilde{\chi}}(\omega) \hat{h}(\omega)-\widehat{\chi}_{d}(\omega)\right|^{2} .
$$

Then, we obtain (12) by substituting in (28) the equalities $\hat{\chi}=\frac{\hat{p}}{\hat{q}} \hat{\varphi}, \hat{a}_{\chi}=\left|\frac{\hat{p}}{\hat{q}}\right|^{2} \hat{a}_{\varphi}, \widehat{\tilde{\chi}}=\widehat{\tilde{\varphi}} \cdot \widehat{\beta_{+}^{N-1}}$ with $\widehat{\beta_{+}^{N-1}}(\omega)=$ $\hat{d}^{N}(\omega) /(j \omega)^{N}, \hat{q}=\hat{h} \cdot \hat{d}^{N}$.

\section{REFERENCES}

[1] M. Unser, "Splines: A perfect fit for signal and image processing," IEEE Signal Process. Mag., vol. 16, no. 6, pp. 22-38, Nov. 1999.

[2] G. Kindlmann and J. W. Durkin, "Semi-automatic generation of transfer functions for direct volume rendering," in Proc. of the IEEE Symposium on Volume Visualization (VVS), 1998, pp. 79-86.

[3] P. Thévenaz, T. Blu, and M. Unser, "Interpolation revisited," IEEE Trans. Med. Imag., vol. 19, no. 7, pp. 739-758, Jul. 2000.

[4] M. Unser, "Sampling-50 Years after Shannon," Proc. IEEE, vol. 88, no. 4, pp. 569-587, Apr. 2000.

[5] T. M. Lehmann, C. Gönner, and K. Spitzer, "Survey: Interpolation methods in medical image processing," IEEE Trans. Med. Imag., vol. 18, no. 11, pp. 1049-1075, Nov. 1999.

[6] E. Meijering, W. Niessen, and M. Viergever, "Quantitative evaluation of convolution-based methods for medical image interpolation," Medical Image Analysis, vol. 5, no. 2, pp. 111-126, Jun. 2001.

[7] T. Blu, P. Thévenaz, and M. Unser, "MOMS: Maximal-order interpolation of minimal support," IEEE Trans. Image Process., vol. 10, no. 7, pp. 1069-1080, Jul. 2001.

[8] _ "Linear interpolation revitalized," IEEE Trans. Image Process., vol. 13, no. 5, pp. 710-719, May 2004.

[9] L. Condat, T. Blu, and M. Unser, "Beyond interpolation: Optimal reconstruction by quasi-interpolation," in Proc. of IEEE ICIP, vol. 1, Sep. 2005, pp. 33-36.

[10] I. Selesnick, "Maximally flat low-pass digital differentiators," IEEE Trans. Circuits Syst. II, vol. 49, no. 3, pp. 219-223, Mar. 2002.

[11] H. Kirshner and M. Porat, "On derivative approximation from sampled data," in Proc. of SampTA, Jun. 2007.

[12] M. J. Bentum, T. Malzbender, and B. B. Lichtenbelt, "Frequency analysis of gradient estimators in volume rendering," IEEE Trans. Vis. Comput. Graphics, vol. 2, no. 3, pp. 242-254, Sep. 1996

[13] M. Unser and J. Zerubia, "A generalized sampling theory without bandlimiting constraints," IEEE Trans. Circuits Syst. II, vol. 45, no. 8, pp. 959-969, Aug. 1998.

[14] S. Ramani, D. Van De Ville, T. Blu, and M. Unser, "Nonideal sampling and regularization theory," IEEE Trans. Signal Process., vol. 56, no. 3 , pp. 1055-1070, Nov. 2008.

[15] M. E. Goss, "An adjustable gradient filter for volume visualization image enhancement," in Proc. of Graphics Interface, 1994, pp. 67-74.

[16] T. Theuß1, H. Helwig, and E. Gröller, "Mastering windows: improving reconstruction," in Proc. of the IEEE Symposium on Volume Visualization (VVS), 2000, pp. 101-108.

[17] T. Möller, R. Machiraju, K. Mueller, and R. Yagel, "Evaluation and design of filters using a Taylor series expansion," IEEE Trans. Vis. Comput. Graphics, vol. 3, no. 2, pp. 184-199, April-June 1997.

[18] G. Strang and G. Fix, "A Fourier analysis of the finite element variational method," in Constructive Aspects of Functional Analysis, Cremonese, Rome, Italy, 1971, pp. 796-830.

[19] T. Blu and M. Unser, "Quantitative Fourier analysis of approximation techniques: Part I-interpolators and projectors," IEEE Trans. Signal Process., vol. 47, no. 10, pp. 2783-2806, Oct. 1999.

[20] M. Jacob, T. Blu, and M. Unser, "Sampling of periodic signals: A quantitative error analysis," IEEE Trans. Signal Process., vol. 50, no. 5, pp. 1153-1159, May 2002.

[21] L. Condat and D. Van De Ville, "Quasi-interpolating spline models for hexagonally-sampled data," IEEE Trans. Image Process., vol. 16, no. 5, pp. 1195-1206, May 2007.

[22] M. Unser and T. Blu, "Cardinal exponential splines: Part I-Theory and filtering algorithms," IEEE Trans. Image Process., vol. 53, no. 4 , pp. 1425-1438, Apr. 2005

[23] S. Mallat, A wavelet tour of signal processing. Academic Press, 1999.

[24] M. Unser and T. Blu, "Wavelet theory demystified," IEEE Trans. Signal Process., vol. 51, no. 2, pp. 470-483, Feb. 2003. 
[25] U. R. Alim, T. Möller, and L. Condat, "Gradient estimation revitalized," IEEE Transactions on Visualization and Computer Graphics (Proc. of IEEE Visualization 2010), vol. 16, no. 6, Nov.-Dec. 2010, to appear.

[26] D. den Hertog, R. Brekelmans, L. Driessen, and H. Hamers, "Gradient estimation schemes for noisy functions," Tilburg University, Center for Economic Research, Tech. Rep. 12, 2003.

[27] Y. C. Eldar and M. Unser, "Nonideal sampling and interpolation from noisy observations in shift-invariant spaces," IEEE Trans. Image Process., vol. 54, no. 7, pp. 2636-2651, Jul. 2006. 